\title{
Désordre et légitimité du politique en Afghanistan
}

\author{
Gilles Dorronsoro
}

\section{OpenEdition}

\section{Journals}

\section{Édition électronique}

URL : http://journals.openedition.org/conflits/155

DOI : 10.4000/conflits. 155

ISSN : 1777-5345

Éditeur :

CCLS - Centre d'études sur les conflits lilberté et sécurité, L'Harmattan

Édition imprimée

Date de publication : 15 mai 1997

ISSN : 1157-996X

Référence électronique

Gilles Dorronsoro, "Désordre et légitimité du politique en Afghanistan », Cultures \& Conflits [En ligne], 24-25 | hiver 1996- printemps 1997, mis en ligne le 15 mars 2006, consulté le 30 mars 2021. URL: http://journals.openedition.org/conflits/155; DOI : https://doi.org/10.4000/conflits.155

Ce document a été généré automatiquement le 30 mars 2021.

Creative Commons License 


\title{
Désordre et légitimité du politique en Afghanistan
}

\author{
Gilles Dorronsoro
}

1 Les perceptions changeantes du conflit afghan depuis plus d'une quinzaine d'années sont révélatrices des nouvelles grilles d'analyse des relations internationales, autant que des évolutions internes au conflit. Pendant l'occupation soviétique (1980-1989), la guerre est généralement interprétée dans le cadre de l'affrontement est-ouest. Même si le concept d'islamisme a été construit à partir d'observations faites notamment sur le terrain afghan ${ }^{1}$, les acteurs locaux et leur stratégies sont surtout pris en compte par rapport aux enjeux globaux de la rivalité américano-soviétique. Dans cette perspective, toutes les actions sont décryptées comme si elles avaient un sens dans ce jeu global. Les analyses laissent peu de place au hasard, à l'autonomie des acteurs locaux ou aux jeux bureaucratiques pour expliquer les prises de décisions. Par exemple, l'invasion soviétique est replacée dans le cadre d'une grande stratégie de conquête des mers chaudes, en continuité avec les objectifs de l'Empire russe ${ }^{2}$. La pensée géopolitique, en particulier quand elle suggère de fausses analogies historiques ${ }^{3}$, est ici particulièrement opérante dans cette recherche à tout prix d'une cohérence générale des événements. Après le retrait soviétique (1989), et contrairement à la plupart des prévisions, les moudjahiddin se révèlent incapables de renverser le régime prosoviétique de Nadjiboullah ${ }^{4}$. La crise afghane, ramenée à des dimensions régionales, suscite des interrogations sur la "vraie nature" du conflit. Les remises en cause, sur le ton du retour au réalisme, s'accélèrent après 1992, quand la chute du régime de Kaboul débouche sur une nouvelle guerre civile entre les partis vainqueurs et aboutit à la destruction de la capitale, partagée entre factions adverses. Dès lors, les interprétations dominantes reflètent des hypothèses sur la nature non-politique du conflit : la guerre est ramenée à des facteurs ethniques ou tribaux, qui sont de l'ordre du culturel, au sens pratiquement génétique du terme. Le politique (idéologie, partis) n'est donc plus considéré comme un facteur pertinent d'explication des comportements, les stratégies sont, à la limite, inexistantes. C'est le retour au naturel du (mauvais) sauvage, il n'y a plus guère à expliquer, reste à déplorer la propension des Afghans à la lutte interne. Dans une optique proche, l'Afghanistan est décrit comme une situation hobésienne ${ }^{5}$, 
un cas limite où la lutte de tous contre tous ne donne plus véritablement prise à une analyse politique, le chaos, l'aléatoire, empêchant toute mise en ordre des événements, qui deviennent pur récit. La guerre afghane s'intégre alors dans le discours classique des relations internationales comme un exemple de désordre, qui remet en cause l'état initial du système. Dans cette perspective systémique (et donc conservatrice), le désordre correspond à la rupture d'un équilibre et, puisque le système international est l'élément de référence, la perturbation est définie par rapport à lui, sans prendre en compte les perceptions des acteurs au niveau local. La géopolitique traditionnelle, et plus généralement la pensée réaliste, en donnant une définition objectivante des situations, propose donc des critères extérieurs aux acteurs pour définir le désordre. Le fondamentalisme dans les pays musulmans, les tensions inter-étatiques à la frontière indo-pakistanaise ou la guerre civile au Libéria sont indistinctement qualifiés de désordre. Après la dislocation de l'URSS et la multiplication rapide des discours globalisant, les conflits "périphériques" sont ainsi présentés comme un facteur de désordre par rapport au monde en équilibre rêvé par une certaine diplomatie. Les crises qui ne sont plus intégrables dans une logique globale deviennent des curiosités à interpréter sur le mode exotique et infra-politique de l'ethnicité ou des intérêts économiques de quelques mafias. Ces dicours relèvent du normatif et on peut surtout s'interroger sur leur fonction institutionnelle, plus que sur leur pertinence à décrire le réel. Il faut, selon nous, rompre avec cette approche objectiviste et replacer les perceptions subjectives des acteurs au centre de l'analyse. En fait, loin d'être une plongée dans un chaos incompréhensible, la guerre après la chute de Kaboul (avril 1992) obéit d'abord à une logique politique de crise : un affrontement violent autour d'enjeux relativement bien définis et une lutte implacable pour la survie, qui exclut largement tout compromis autre que tactique. Pour nous, cette situation ne relève pas $\mathrm{du}$ réflexe culturel, mais de la lutte pour le pouvoir. Les partis politiques poursuivent des stratégies, ont des objectifs et se construisent comme des forces de plus en plus organisées : le phénomène majeur de ces dernières années est bien la reconstruction progressive d'un système politique centralisé. Contrairement à une perception répandue, la carte politique afghane s'est en effet continuellement simplifiée depuis le retrait soviétique car la concurrence entre les acteurs politiques a eu pour effet d'éliminer les plus faibles, ce qui a entraîné mécaniquement une diminution du nombre des acteurs ${ }^{6}$. D'une dizaine de partis principaux contrôlant des groupes sans continuité territoriale, on est passé aujourd'hui des territoires politiques étendus. La taille croissante des acteurs politiques a également permis un saut qualitatif dans les formes de combat. La guérilla a fait place à des engagements de type plus classique qui ont pour enjeu le contrôle des villes. L'utilisation de l'arme aérienne et de blindés va aujourd'hui de pair avec des concentrations de plusieurs milliers d'hommes. Ces modifications des formes de combats ont rendu possibles des percées spectaculaires, au moins pour la prise des villes. Tenir les montagnes n'est plus un gage de sécurité, mais le signe d'une marginalisation. Le futur système politique est l'enjeu des combats entre les mouvements. Les retournements d'alliance ne se sont pas faits au hasard, mais en fonction des possibilités pour les partis de survivre ou non dans le futur Etat ${ }^{7}$. De 1992 à 1994, deux phénomènes expliquent, pour l'essentiel, les combats et les renversements d'alliance. D'une part, le Hezb-é islami et du Jamiat, proches idéologiquement car issus de la même organisation islamique dans les années soixante-dix, se battent pour le contrôle de la capitale et la reconstruction d'un Etat centralisé et islamique. D'autre part, le Hezb-é wahdat, qui regroupe des chi'ites de l'ethnie hazara, et le Djoumbesh, 
qui développe un discours ethno-nationaliste ouzbek ${ }^{8}$, tentent de peser sur les futurs équilibres politiques pour garantir des formes d'autonomie régionale que refusent les autres partis. Ces deux mouvements chercheront donc à éviter qu'une coalition soit suffisamment forte pour éliminer l'autre définitivement et puisse ensuite reconstruire un pouvoir central fort. L'apparition du mouvement des Taléban et sa rapide extension va radicalement changer les systèmes d'alliance. A l'été 1994, un groupe d'étudiants en religion (Taléban) lance une attaque contre les commandants qui tiennent Spin Boldak, un poste à la frontière afghano-pakistanaise sur la route Quetta-Qandahar. Quelques semaines après le succès de cette première opération, les Taléban prennent Qandahar (octobre 1994) puis Hérat (septembre 1995), Djalalabad et enfin Kaboul (septembre 1996). Pour s'opposer à la progression des Taleban, les anciens partis de la résistance (Jamiat, Hezb-é islami, Ettehad, Hezb-é wahdat), s'allient aux ex-milices gouvernementales (Djoumbesh). Cette bipolarisation, accélérée par la chute de Kaboul aux mains des Taléban laisse entrevoir pour la première fois la fin possible des combats par une victoire militaire. Le comportement des acteurs politiques s'inscrit donc dans une rationalité particulièrement contraignante, mais les alliances faites au nom de considérations tactiques, pour survivre, ont finit par créer le sentiment d'une politique purement instrumentale. De plus, l'oppression de la population par les partis politiques a mis fin à l'unanimité des premières années de combat contre un gouvernement athée, soutenu par une invasion étrangère. Cette situation injustifiable de désordre et d'injustice, a ainsi favorisé l'émergence des Taleban, qui étaient en quelque sorte attendus par la population. Après avoir décrit les rapports entre population civile et groupes armés, nous verrons l'évolution de la perception du politique, puis l'émergence des Taleban comme réponse au désordre.

2 Les commandants et la population.

3 Dans les premiers temps de la guerre, il n'y a pas de distinction entre civils et combattants, tout homme disposant d'une arme étant engagé (au moins à la campagne) contre le gouvernement et l'armée soviétique. La séparation progressive qui s'est installée entre les moudjahiddin (Combattants de la Foi) et les civils est liée à la professionnalisation des combattants, conséquence de la confrontation avec une armée moderne effectuant des opérations de grande envergure qui rendent périmées les techniques classiques de la guérilla tribale. Cette distinction militaire-civil fut plus ou moins totale et rapide selon les endroits mais, en général, un seuil a été franchi vers 1986, en raison de l'aide extérieure qui arrive alors de façon massive9. Les groupes de combattants, regroupés progressivement dans des lieux spécialisés (qarargah), vivent entre eux, souvent en dehors du village, dans une relation d'extériorité avec la population, accrue par la mobilité géographique croissante des moudjahiddin. Dans les endroits peu touchés par la guerre, le modèle initial de non-différentiation entre population et combattants a survécu et la mosquée sert encore de lieu de coordination des hommes en armes. Par ailleurs, une différentiation intervient à l'intérieur des groupes. Certains armements (anti-tanks, mortiers par exemple) nécessitent une formation minimale qui empêche une interchangeabilité parfaite des combattants, alors que de nouvelles tactiques, plus élaborées, obligent à une coordination effective au sein des groupes. Chez Massoud, par exemple, on a très tôt l'apparition de moudjahiddin à plein temps, organisés sur le modèle d'une armée régulière ${ }^{10}$. Enfin, et surtout, une structure hiérarchique apparaît: les commandants (chefs de groupe) acquièrent le statut d'autorité politique et militaire locale et deviennent les 
interlocuteurs et les représentants de la population. Or, il est possible, au moins en première analyse, de considérer les commandants comme des unités politiques relativement autonomes. En effet, les partis ne sont pas une source de légitimité essentielle pour les commandants, dont l'acceptation par la population repose, le plus souvent, sur une implantation locale préalable. Sans évacuer toute dimension idéologique, la relation parti-commandant repose d'abord sur la fourniture d'armement. L'autonomie des commandants, qui peuvent facilement changer de parti, installe un système sans autorité régulatrice. En conséquence, les affrontements entre groupes, parfois du même parti, sont une constante depuis le début de la guerre et leur aggravation est patente après le retrait des Soviétiques, dont la présence était un facteur d'union. Les relations entre commandants sont marquées par la concurrence qui naît de rivalités personnelles, de questions logistiques, du contrôle d'endroits stratégiques, de l'aide humanitaire, et il n'y a pas d'instance régulatrice qui puisse imposer une norme de comportement et garantir les transactions. Les partis, euxmêmes en concurrence, ne peuvent établir de règle du jeu entre les commandants. Les jirga (Conseil tribal) dans certaines régions tribalisées, ou les oulémas, comme à Qandahar pendant un temps, peuvent jouer un rôle d'arbitrage, mais ces instances ont un rôle de conseil et interviennent surtout après le déclenchement des conflits, pour trouver un compromis. La concurrence débouche donc fréquemment sur des affrontements armés. La plupart des commandants importants ont été victimes de tentatives d'assassinat et un nombre considérable ont été contraints au ralliement, à l'exil ou assassinés ${ }^{11}$. La coopération est limitée à des actions ponctuelles, chacun ayant d'abord le souci de préserver son autonomie. Les shoura (Conseil) qui se sont multipliées après 1989, ont rarement un rôle concret et seuls des systèmes plus intégrés, où une organisation encadre étroitement les commandants, peuvent établir une coopération (la Shoura-yé nazar de Massoud dans le nord-est, par exemple). Ajoutons que les conflits entre commandants sont loin d'être marginaux, même avant 1992 et peuvent d'ailleurs apparaître entre commandants du même parti, particulièrement si celui-ci est dominant dans une région, empêchant le jeu normal des différentiations. Ainsi, dans l'ouest, en 1988, les combats entre deux commandants du Harakat-é enqelâb de l'Helmand, mollah Nassim et "Raïs" Abdoul Wahed, ont fait des centaines de morts, provoquant l'interruption momentanée de la circulation des convois entre le Pakistan et la région d'Hérat. De même, au Badkhashan, les combats entre Nadjmouddin et Bassir Khan divisent la province depuis plusieurs années.

4 La violence qui naît de cette concurrence n'est pas gérable socialement dans les procédures existantes. En effet, l'affiliation à un commandant (plus qu'à un parti) fonctionne comme un réseau de solidarité (qawm) ${ }^{12}$ : le politique est vécu sur le mode de l'appartenance familiale. Ainsi l'adhésion politique crée des solidarités en partie autonomes des anciennes solidarités territoriales, tribales ou religieuses. En conséquence, il ne peut pas y avoir de purge ${ }^{13}$ ou d'élimination dans un groupe ou un parti sans déclenchement d'une vendetta. Mais il n'y a pas non plus de régulation possible des conflits par le système de responsabilité collective et de vengeance. Les armements en jeu (et donc le nombre de morts potentiels) et les niveaux multiples de solidarité, qui font passer les conflits du niveau local au niveau national et inversement, déséquilibrent le système. La vendetta, qui a d'abord une fonction de dissuasion, ne fonctionne plus comme régulateur social, mais généralise la violence ${ }^{14}$. On peut distinguer, d'après leur origine, plusieurs types de conflits entre commandants. Tout d'abord, les oppositions entre partis au niveau national peuvent 
entraîner des affrontements locaux. Par exemple, le conflit entre le Hezb-é islami et le Jamiat a été une source constante de friction. Le Hezb-é islami a essayé de rompre les lignes de communication de Massoud, y compris en passant des accords avec le gouvernement, alors que Massoud poursuivait son expansion dans le nord-est largement au détriment du Hezb-é islami, bien implanté dans ces provinces au début de la guerre. En 1990, le conflit a débouché sur une affaire particulièrement grave quand un commandant du Hezb-é islami, probablement sur ordre direct de son chef, Hekmatyar, a tendu une embuscade à des cadres de Massoud, les capturant puis les exécutant ${ }^{15}$. Dans une autre région, au Hazarajat, la lutte entre les partis chi'ites révolutionnaires pro-iraniens et conservateurs, entre 1982 et 1986 pour l'essentiel, a causé des destructions importantes dans une zone par ailleurs épargnée par les forces soviéto-gouvernementales. Ensuite, les mobilisations se faisant souvent sur la base d'un réseau de solidarité (qawm), les commandants se trouvent parfois héritiers d'antagonismes communautaires anciens. Certains affrontements ont donc une origine communautaire, même si la réinterprétation politique des oppositions communautaires peut en changer profondément la dynamique, et notamment le règlement. D'autres conflits, enfin, ne renvoient pas à des oppositions communautaires ou politiques, mais à un jeu très local d'affrontements pour le pouvoir. La ville de Tashqourghan ${ }^{16}$, sur la route entre Kaboul et Mazar-é Sharif offre un exemple de ces situations de concurrence violente débouchant sur des vendetta. Dans cette ville, les conflits entre commandants ont été permanents depuis le début de la guerre. Le bazar est divisé entre différentes zones contrôlées par les commandants et un moudjahidin ne peut guère aller dans une zone appartenant à un autre parti. Manifestation concrète de cette hostilité, les commandants ont élevé des tours fortifiées avec des murs de deux mètres d'épaisseur et des meurtrières pour les tireurs embusqués. Les déplacements de nuit dans le bazar sont dangereux en raison des embuscades. Pendant l'été 1991, la ville a vu l'assassinat de son principal commandant, mohallem Razorq, par Shakâr "Papashâ", lui même tué le lendemain. A l'intérieur même des partis les affrontements sont parfois violents ${ }^{17}$. Contrairement à d'autres régions, il n'y a pas ici de facteurs sociaux ou historiques véritablement explicatifs de ces antagonismes. L'origine sociale des principaux commandants n'est pas un facteur de division: les notables terriens, bây, ont gardé à Tashqourghan l'essentiel de leur pouvoir, il n'y a pas d'affrontement avec de nouvelles catégories sociales, même si la montée des éduqués peut être partiellement explicative des tensions. Les oulémas ont, certes, gagné de l'influence, mais ils restent à l'écart du pouvoir ${ }^{18}$. Le facteur ethnique, s'il permet en partie de comprendre la structure partisane, n'explique pas les affrontements. En effet, la ville est majoritairement tâdjik, avec une minorité importante d'Ouzbeks et de Pashtounes, mais il ne semble pas y avoir de base ethnique très marquée pour les commandants. Tashqourghan montre, selon nous, que les conflits ne s'expliquent pas toujours par des tensions communautaires, qui renverraient à la structure sociale. La concurrence entre les commandants est ici le facteur décisif qui explique les combats, le politique s'explique par le politique : la lutte pour le pouvoir ne renvoie qu'à elle-même ${ }^{19}$.

5 Dans leurs relations avec la population, les commandants ne rentrent pas véritablement dans un rapport de négociations, qui les obligerait à une prise en compte des demandes sociales. En effet, l'aide extérieure et une force militaire leur donne les moyens d'imposer leurs décisions sans véritable discussion. (Il faut ici nuancer car les pratiques de pouvoir sont plus ou moins coercitives selon les individus et les régions.) La population n'est pourtant pas totalement dépourvue de moyens de pression sur les 
commandants. Ceux-ci restent ainsi tenus par leur appartenance à des réseaux de solidarité qui constituent leur base sociale (famille élargie par exemple), mais cette protection ne s'étend pas à toute la population. L'intégration des moudjahiddin peut également se faire par le biais de mariages dans les familles dominantes. De même, la corruption est une autre façon de créer des obligations et donc de rendre prévisible les réactions des moudjahiddin, ce qui évite l'arbitraire total et la violence physique. Parfois, des notables et des oulémas ont été délégués auprès des commandants pour demander la fin des combats, par exemple au Hazarajat en 1990, quand la guerre interpartis était devenue par trop destructrice. La protestation est quelquefois publique : en 1992, dans le bazar de Baraki-Barak, les commerçants affichent des posters du Hezb-é islami pour manifester leur opposition au commandant local du Jamiat. De façon prévisible, les situations de violence maximale se trouvent dans les cas où les moudjahiddin opèrent en dehors de leur village ou de leur région d'origine, dans les villes notamment. Ainsi, des scènes de violence ont fréquemment eu lieu à Kaboul entre 1992 et 1994, notamment la mise à sac des quartiers Hazaras par les hommes de Massoud, les exécutions collectives de chi'ites par Sayyaf ou les viols et les pillages à grande échelle par les hommes de Dostum ${ }^{20}$. La méfiance initiale des couches urbaines à l'égard des moudjahiddin s'est ainsi trouvé renforcée après la prise des villes en 1992. D'autre part, les moudjahiddin appelés en renfort par leur parti dans une autre région se rendent parfois coupables d'actes de pillage, d'où les heurts entre les moudjahiddin locaux et extérieurs. C'est notamment ce qui a provoqué des conflits au sein du Hezb-é islami dans le Logar et à Ghazni en $1994^{21}$. La violence dans les rapports quotidiens est probablement le trait marquant des relations entre moudjahiddin et population. Les commandants abusent souvent de leur pouvoir, leur brutalité les font craindre et ils n'hésitent parfois pas à faire assassiner leurs opposants ou des concurrents potentiels, créant une atmosphère de peur et d'humiliation. Affichant avec arrogance les signes extérieurs de leur position, notamment les Pajero ou les Toyota (double cabine) à vitres fumées, ils deviennent des caricatures de potentats locaux., toujours entourés de leurs gardes du corps. Leurs rapports avec les anciens notables, qui gardent souvent une forte reconnaissance sociale, sont souvent exécrables. Dans certains cas limites (Khédir au Hazarajat), les commandants n'ont aucune légitimité et ne se maintiennent que par la force armée, comme une troupe d'occupation. Le respect de la sphère privée, un des grands revendications de la révolte anticommuniste de 1978, est remis en cause par l'attitude de certains moudjahiddin ${ }^{22}$. Un nombre important de commandants volent et pillent les villages tombés sous leur domination. La rupture avec l'ordre social est à son point maximal quand les commandants pénétrent dans les maisons, particulièrement dans le monde pashtoune où la notion d'honneur familial (nang) est centrale ${ }^{23}$ : les viols et les rapts de femmes sont des atteintes sociales très graves qui engagent l'ensemble du groupe familial et ses alliés. Par ailleurs, les possibilités juridiques d'obtenir réparation des torts causés par les commandants sont en pratique très limitées. Les oulémas, en temps que juges (qazi), seraient normalement les garants de l'autonomie de la sphère du droit, inspiré de la chariat. Mais, l'arbitraire des commandants s'arrêtent peu à ces considérations. Dans une même région, les partis (voire les commandants) développent chacun leur propres réseaux de juges islamiques, ce qui rend évidemment l'indépendance de la justice très aléatoire. Signe de la puissance croissante des commandants, le poids des oulémas comme juge semble avoir décliné dans les années quatre-vingts ${ }^{24}$. 
6 Le commandant, reprenant en cela le rôle traditionnel du notable (khan) dans la société afghane, se pose en intermédiaire entre la communauté et l'extérieur ${ }^{25}$. Il utilise sa position de médiateur pour en tirer un profit personnel, généralement financier. La plupart des commandants, qu'on peut appeler patrimoniaux, ne font pas de distinction claire entre leur personne et leur rôle de représentant d'un parti politique. Au niveau local, le commandant est bien souvent à la tête d'une entreprise de type familiale : le frère succède au frère, les postes sont attribués systématiquement à la famille élargie. Les dons du parti au commandant sous forme d'armes ou d'argent sont utilisés à des fins personnelles, tout comme l'aide humanitaire. Nombre de commandants ont ainsi amassé un capital considérable, réinvesti dans la terre ou les entreprises commerciales au Pakistan ou ailleurs. Ces comportements patrimoniaux, contrairement à une idée admise, ne sont pas forcément considérés comme normaux par la population ${ }^{26}$. De plus, en installant des postes sur les routes, les commandants se procurent des rentrées d'argent et contribuent au renchérissement des marchandises, pour la plupart importées, sans droit de douane, des pays voisins. De ce fait, les moudjahiddin sont perçus comme parasitaires à partir du moment où ils ne combattent plus le gouvernement et n'organisent pas une administration locale. Or, après le retrait soviétique, le gouvernement garde une position globalement défensive et une proportion importante du territoire n'est plus touchée par les combats. Les moudjahiddin, généralement peu mobile du fait de la segmentation politique, ne combattent plus qu'en minorité. De plus, et sauf exception, le travail d'administration des commandants reste très faible en dehors de l'apport des ONG (Organisation Non Gouvernementale).

7 Ces situations de désordre ne sont pas répartie de façon aléatoire sur tout le territoire. En effet, en raison des différences d'organisation sociale, les moudhahiddin ne s'organisent pas partout de la même façon. Dans les régions non-tribalisées, des embryons d'administration fonctionnent sur le modèle étatique avec l'instauration de zone de stabilité régionales. Les exemples les plus caractéristiques sont ceux de Massoud, celui de Dostum et Ismael Khan dans l'ouest (jusqu'en 1994). Ces organisations régionales mobilisent éventuellement sur des sentiments communautaires, mais utilisent toujours à un discours politique. Cette situation correspond à la domination politique d'un groupe social, les éduqués ${ }^{27}$, possible en raison d'une moins grande fragmentation sociale. L'absence de tribalisation, caractéristique du sud pashtoune, et une moins grande différence ville-campagne, facilite en effet l'intégration des éduqués en milieu rural. Cependant, si le nord se territorialise plus vite, des provinces comme Koundouz ou Baghlan connaissent une situation de fragmentation politique forte, alors que Ghazni, au sud, connaissait une certaine stabilité dès 1992, mais sous la direction d'un groupe d'oulémas. Au sud nous avons, au contraire, le maintien d'une division politique plus grande, qui s'explique par une fragmentation sociale limitant le jeu des partis, qui correspondent plus étroitement à des réseaux de solidarités préexistants. Dans ces conditions, les éduquées ne parviennent pas à s'imposer comme leaders locaux et surtout à étendre progressivement leurs zones de pouvoir comme ils le font au nord. Dans les régions segmentaires (principalement au sud), la carte politique est souvent plus complexe, les oppositions entre segments tribaux empêchant l'émergence de pouvoirs centralisés, ce qui amène parfois une véritable anarchie; comme à Qandahar. Entre 1992 et 1994, le partage de Qandahar entre différents commandants a crée une situation de désordre exceptionnelle. Pendant deux ans, les commandants n'ont assuré aucune 
administration de la ville et, surtout, ont fait régner une atmosphère d'insécurité permanente. Le comportement des moudjahiddin dans la ville était alors ouvertement déviant : musique indienne dans toute la ville, combats de chien, accrochages fréquents entre groupes pour des motifs futiles ${ }^{28}$, haschich et opium fumés en public, absence de toute administration, prostitution masculine et féminine, vidéo $\mathrm{X}$ facilement disponible dans le bazar... Pour l'étranger de passage, l'atmosphère de la ville rappelle alors immanquablement un western. Economiquement, la ville est asphyxiée en raison de l'insécurité. Le commerce est contrarié par les nombreuses taxes prélevées sur la route, les vols de camions... De plus, les combats, fréquents dans le bazar, empêchent tout effort de reconstruction. Les personnalités religieuses qui pouvaient faire le lien entre les commandants et la population, comme pir Murtch Agha, étaient clairement dépassées par la situation ${ }^{29}$ et le contrôle des états-majors des partis politiques tout aussi limité. Les demandes de familles victimes de moudjahiddin demeuraient donc sans suite. Le comportement des moudjahiddin n'est pas sans rappeler celui des bandes de pây loutch, la confrérie de mauvais garçons de Qandahar, qui présente des similitudes avec celles des kôkâ à Kaboul, des louti (de lout : voyou) en Iran et des chabab à Tripoli (Liban) ${ }^{30}$. Cette bande rassemblait des jeunes gens du bazar, souvent fils de bouchers, aubergistes... toutes professions en général peu prestigieuses. Les signes distinctifs d'appartenance étaient le grand couteau, l'habit jaune et les babouches. Les pâylouch se devaient également de respecter un code d'honneur et de solidarité entre membres et constituaient donc une forme de réseau de solidarité (qawm). Ils étaient connus pour aimer les combats de chiens qu'ils organisaient souvent un peu en dehors de la ville à côté d'un lieu de pélerinage connu, près duquel ils fumaient du haschisch en grande quantité31 . Ils étaient aussi chargés d'assurer la sécurité de certaines rues (probablement une forme de racket), et servaient, à l'occasion, de tueurs à gage. Au moment de l'invasion soviétique (Qandahar ne bouge pas avant), les pâylouch se mobilisent massivement derrière leur chef pour participer aux combats contre l'occupant. Il n'y a pas de continuité directe entre ces bandes et les moudjahiddin, mais reprise des mêmes pratiques déviantes (haschisch, combats de chiens...). Depuis des années (au moins depuis le retrait soviétique), ce désordre est au centre des préoccupations de la population. En règle générale, le discours que l'on entend depuis des années dans les auberges (tchaikhana) est très critique des commandants et de leurs comportements arbitraires. Ainsi, l'écoute du discours des Afghans conduit notamment à remettre en question l'interprétation culturaliste, qui fait du conflit armé et de l'absence d'Etat une donnée "naturelle" de la vie sociale des Afghans.

Mobilisation populaire et discours sur le politique.

9 La mobilisation unanime ${ }^{32}$ de la population contre le régime, caractéristique des premières années de guerre, a évolué vers une situation beaucoup plus complexe, où le jihad n'est plus l'évidence de l'engagement. Au début de la guerre (1978-79), les soulèvements sont provoqués par des revendications locales et un rejet moral de l'autorité politique, puis les discours s'unifient par la référence religieuse, qui impose un sens légitime au combat. Désormais, la référence à l'Islam oriente les comportements individuels : le jihad est vécu comme une nécessité personnelle et un devoir religieux ${ }^{33}$, les notions de moudjahidin et de shahid ${ }^{34}$ sont au centre du discours des individus sur le sens de leur engagement ${ }^{35}$. Conformément à une longue tradition, les grands combattants morts au combat deviennent l'objet d'un culte populaire, comparable à celui des pir: "dans l'Afghanistan d'avant-guerre, les martyrs ne se 
distinguaient pas des autres saints hommes dont on visitait les tombeaux" ${ }^{16}$. Ainsi, dans certaines mosquées d'Hérat, on prie encore pour les martyrs des guerres contre les Britanniques au XIXe siècle. Souvent, un Front garde le nom de son premier commandant et se place ainsi sous son patronage. Les exploits des grands moudjahiddin morts au combat sont chantés et leurs posters circulent dans tout le pays. Par ailleurs, la mémoire historique fait apparaître l'Islam comme une légitimation des révoltes populaires contre l'envahisseur kafir (Britanniques au XIXe siècle) ou l'Etat (1929), le religieux étant, on l'a vu, un modèle pour la contestation du pouvoir en pays musulman. De plus, la Révolution iranienne voisine n'a pas eu de retentissement que chez les chi'ite et présentait pour les intellectuels un modèle contemporain de révolution religieuse. Dans cette phase initiale, il n'y a pas contradiction entre référence à l'Islam et au sentiment national. Avec l'arrivée des Soviétiques (décembre 1979), les parallèles historiques entre Babrak Kârmal et Shâh Shoujâa ${ }^{37}$ sont monnaie courante dans les tracts appelant à la résistance contre l'occupant. Au niveau individuel, la notion religieuse de moudjahidin et de shahid fait écran à une perception politique de la lutte, puisque le jihad est une relation entre l'Homme et Dieu et non entre deux adversaires. En 1978-79, la mobilisation populaire contre le gouvernement communiste ne s'est pas faite pas au nom d'une idéologie politique, les militants sont rares au sein de la population rurale et, au sens strict, leur discours idéologique (y compris islamiste) n'est pas compréhensible par une population paysanne très faiblement alphabétisée et politisée. Les partis politiques ne se sont faits connaître ou n'ont été créés qu'après le soulèvement, ils ne l'ont pas initié mais exploité. La population était donc mobilisée initialement sur l'idée de jihad, qui n'est pas de l'ordre du politique mais du religieux. Tout le travail des organisations politiques pour tenter de donner un contenu idéologique à cet engagement a échoué dans une large mesure, en dehors du milieu très minoritaire des éduqués. L'idéologie n'est pas une source significative d'engagement des acteurs. En effet, beaucoup sont ignorants du discours politique qui est incompréhensible ( $95 \%$ d'analphabètes environ).

Après le retrait soviétique, la stratégie défensive du gouvernement porte ses fruits, en particulier dans les milieux urbains. L'Etat cherche notamment à replacer les conflits dans un cadre non-religieux, en jouant de la segmentation sociale pour aggraver les divisions des moudjahiddin ${ }^{38}$. L'absence de pression militaire du gouvernement accroit le nombre des conflits entre moudjahiddin. Par ailleurs, la concurrence s'exacerbe entre les commandants qui pensent la victoire proche. Enfin, le consensus idéologique, très fort au début du conflit, est progressivement mis en cause par le durcissement d'une partie des moudjahiddin, travaillés par des mouvements fondamentalistes de type wahhabites ${ }^{39}$. La présence de combattants étrangers, notamment quand ils cherchent à imposer aux Afghans de nouvelles pratiques religieuses, entraîne des réactions de rejet que l'abondance des financements ne suffit pas à dissimuler. L'attitude extrémiste de certains groupes, surtout après le retrait soviétique, remet en question les arrangements de la société dans la guerre, et affaibli la cohésion de la résistance. Ainsi, la violence exercée contre les soldats gouvernementaux qui se rendent est contraire à l'esprit initial du jihad. Par exemple, au printemps 1989, pendant la bataille de Djalalabad, les moudjahiddin du parti de Khalès ont massacré une soixantaine de soldats prisonniers et ont renvoyé les cadavres, coupés en morceaux, au gouvernement. Dès lors, un changement profond intervient dans le discours populaire sur la guerre. Les grilles de lecture qui opposaient communistes athées et moudjahiddin ne sont plus opératoires. Dans l'ordre du discours, un des phénomènes les plus 
surprenants est l'absence apparente de cohérence dans les préférences politiques exprimées ${ }^{40}$. Ainsi, un individu peut exprimer dans le même temps son admiration pour les exploits d'un moudjahiddin et, dans le même temps, sa préférence pour le communiste Nadjiboullah. Le terme de jihad laisse progressivement place à celui de guerre civile (jang-e dakheli). Personne ne décrit plus les affrontements en termes religieux et le soutien populaire aux moudjahiddin est beaucoup plus faible. Par exemple, les services aux combattants deviennent généralement payants ${ }^{41}$. Le terme de moudjahiddin est de plus en plus dévalorisé. Le mot d'oppression, zoulm, est de plus en plus utilisé pour décrire le comportement des commandants, alors qu'il était auparavant réservé au gouvernement. Le terme de gouvernement, hokumat, devient récurrent, les individus regrettent l'absence de gouvernement, de loi, d'ordre civil. Par ailleurs, les modes d'explication des événements politiques sont de type instrumental, centrés sur les ambitions individuelles. Il y a un rejet du politique qui n'est dans l'expérience quotidienne, que la justification de l'intérêt immédiat du plus fort. L'explication ne renvoie généralement à autre chose que la lutte pour la domination. Le politique apparaît comme une sphère d'activité totalement déconnectée de toute moralité et de toute utilité sociale, puisque seul l'intérêt des commandants est pris en compte. Les individus ne peuvent plus interpréter les comportements des commandants et des partis en termes universels, moraux ou idéologiques, la seule explication crédible est purement instrumentale. Les nombreux retournements d'alliance sont ainsi l'expression d'une pure rationalité tactique. Les Taléban ou le refus du politique.

12 Cette situation de désordre a permis l'émergence d'une nouvelle force politique, les Taleban, qui, moins de trois ans après son apparition, occupe les deux-tiers du pays, dont la capitale. Cette progression fulgurante est-elle principalement le résultat d'une aide extérieure? L'implication pakistanaise dans le mouvement est avérée (des combattants penjabis ont été blessés ou faits prisonniers à de nombreuses reprises), de même que le soutien américain, au moins diplomatique ${ }^{42}$. L'appui du Pakistan explique certainement la rapidité avec laquelle le mouvement a pu se constituer en force militaire cohérente, avec des hommes formés au maniement des chars et une efficacité tactique due à la présence d'officiers pakistanais, sans compter la présence de plusieurs centaines de taleban pakistanais. Mais il suffit de rappeler l'échec des Pakistanais dans leur soutien, au moins aussi conséquent, au Hezb-é islami pendant plus de quinze ans pour constater que les Taléban sont un phénomène de grande ampleur, qui dépasse largement la manipulation des services secrets pakistanais (ISI). Pour la première fois, peut-être, les Pakistanais ont joué la bonne carte et se sont trouvés en phase avec un mouvement authentiquement populaire. En effet, l'aide extérieure n'explique pas la grande facilité avec laquelle le mouvement a pu s'étendre pratiquement sans combat dans tout le sud de l'Afghanistan et jouir jusqu'à aujourd'hui d'un fort soutien populaire. Dans toute la phase initiale du mouvement, les combats ont été limités, les commandants se ralliant ou s'enfuyant, sans combattre la plupart du temps. Les commandants qui se sont opposés à l'avancée des Taléban n'ont pas pu compter sur le soutien de la population et beaucoup de leurs propres moudjahidin sont passés à l'ennemi. Le nombre de victimes jusqu'aux premières attaques sur Kaboul doit être de l'ordre de quelques centaines seulement pour le contrôle du tiers de l'Afghanistan. L'impact du discours des Taléban est énorme dans tout le pays, particulièrement dans les régions du sud, les plus fragmentées politiquement et l'espoir populaire a porté les Taléban jusqu'aux portes de Kaboul. L'attente de la population a été un facteur décisif, 
une condition de possibilité du succès des Taléban, qui proposent une solution radicale au désordre. Le succès initial des Taléban est directement lié à une situation locale de désordre : l'émergence du mouvement se fait à Qandahar, la zone d'anarchie la plus notable en Afghanistan, en dehors du courant général de centralisation et de territorialisation que nous avons souligné en introduction. L'histoire des Taleban à ses débuts, telle qu'ils la racontent, part d'un incident qui aurait eu lieu près de Qandahar au printemps 1994. Des voyageurs auraient été victimes d'un commandant, qui aurait notamment violé des femmes. Quoi qu'il en soit de l'exactitude des faits, il y a rupture de deux règles fondamentales du monde afghan et plus particulièrement pashtoune : l'honneur familial et l'hospitalité. Quelques oulémas vivant au Pakistan auraient alors décidé d'intervenir auprès des commandants et devant leur refus auraient pris les armes. Le discours et la pratique des Taléban est d'abord une réponse au désordre par la rupture avec les partis et les commandants, dont certains seront (bien avant Nadjiboullah) pendus publiquement et leur cadavres ornés de billets de banque pour stigmatiser leur corruption. Les Taléban propose un retour au jihad pur des premières années, avec, en plus, un refus des partis politiques qui sont accusés d'être à l'origine de la guerre civile. La légitimité des Taleban est ainsi religieuse et non politique, ce qui explique également son fort impact dans les régions sud, où les partis politiques n'ont pas pu créer les mêmes loyautés qu'au nord. Le mouvement a une dimension charismatique, son leader, mollah Omar, aurait eu des songes, inspirés par Dieu, qui lui aurait commandé de libérer l'Afghanistan de l'anarchie crée par les moudjahiddin. Des rumeurs ont couru sur les pouvoirs magiques des Taléban qui ne seraient pas atteints par les projectiles. Le charisme reconnu à Mollah Omar est le signe de l'attente populaire. Ce dernier n'est pas un ouléma connu, bien qu'il enseigne dans une madrassa au Pakistan (et certains croient qu'il n'est pas ouléma, ce qui prouve que sa légitimité est de l'ordre du charisme plus que dela connaissance). Les grandes familles religieuses d'Afghanistan, les oulémas reconnus, parce qu'ils se sont impliqués dans les jeux politiques n'ont plus la légitimité, la neutralité pour parler au nom de la communauté entière. Les Taléban ne sont pas perçus comme une force politique de plus, mais comme un renouvellement radical de la situation politique. Leur réputation d'honnêteté, l'absence de pillage et de viols en font de véritables moudjahiddin aux yeux du populaire. Ils ont soulevés des espoirs dans la population car ils apparaissaient en dehors du jeu politique. Au-delà, le projet des Taléban est assez simple et propose un retour au sens initial du jihad, qui est essentiellement non-politique. Le slogan simpliste du retour à la chariat est souvent présenté comme une limitation des Taléban alors que ce refus du politique est le fondement le plus sur de son succès populaire. Ce n'est pas hasard si le leader du mouvement Mollah Mohammad Omar a refusé de s'installer à Kaboul après la prise de la ville. Le terme de "Taleban", qui désigne le mouvement, n'est pas un nom de parti, les Taleban n'ont pas d'organisation formelle. La distance affichée par rapport au politique est bien la condition de la légitimité, et donc du succès, d'un religieux investi dans les affaires mondaines. Le titre de Commandeur des Croyants (amir ul mominin) qui a été décerné en 1996 par des oulémas (et non des représentants des partis) à Mollah Omar, est bien le signe de ce refus du politique, la légitimité du leader est d'abord religieuse et s'inscrit pratiquement dans une revendication du Califat. A l'inverse, mawlawi Rabbani, le leader historique du Jamiat, fonde sa légitimité sur son élection (dans des conditions d'ailleurs douteuses) comme président de la République. Le programme des Taléban est centré autour de la recréation de rapports de droit entre les individus. Le ramassage 
des armes, qui est effectué avec une efficacité remarquable, et la libre circulation des hommes et des marchandises, qui leur vaut un soutien constant des grands commerçants du bazar, sont les deux mesures concrètes les plus remarquables. La réparation faite publiquement à un commerçant indou après la prise de Djalalabad vaut comme exemple de ce respect de la propriété privée. Par ailleurs, le pardon est proposé à tous pour leurs activités passées, amnistie (et amnésie) collective qui facilite les ralliements et évite des querelles interminables. Les Taléban sont crédibles dans leur projet de retour à l'ordre car leur mouvement présente deux caractéristiques, en un sens négatives : extériorité par rapport aux appartenances segmentaires, du fait du recrutement religieux, et extériorité par rapport au politique. Le recrutement des cadres du mouvement, qui sont essentiellement des oulémas ou des Taléban, est un gage de neutralité entre les différentes communautés. On retrouve ici la traditionnelle position d'extériorité des oulémas dans les sociétés tribales qui leur permet d'intervenir dans des circonstances exceptionnelles, quand la fragmentation sociale limite la coopération. Des processus semblables ont été observés en particulier chez les Pashtounes du Pakistan (Pathans). Le fait que le mouvement se soit initialement constitué au Baloutchistan pakistanais renforce cette extériorité, de même que le fait que les leaders du mouvement aient été inconnus auparavant. Le recrutement initial dans les madrassas est également un gage de neutralité dans les vendetta locales. Les camps de réfugiés fournissent la première masse de manoeuvre. Dans les régions où ils ont pris le pouvoir, les Taléban ont systématiquement nommés des responsables étrangers à la province. La confiscation des armes et, de façon générale, les opérations de police ne sont donc pas ressenties comme des règlements de compte locaux (et du coup les dénonciations affluent). Les Taléban sont en fait porteurs d'un projet de société qui exclut, au moins en théorie, le politique comme sphère d'activité constituée. Les partis sont compris comme une occasion de division de la société (fitna) ${ }^{43}$, le conflit, même intellectuel, n'est pas perçu comme légitime, le modèle étant la société unifiée des origines de l'Islam. Il y a ici une obligation de consensus qui rappelle à la fois le fonctionnement de la société tribale et des oulémas comme corps social (même si, bien sûr, le conflit reste derrière l'unanimité de façade). Les oulémas doivent nommer le gouvernement, sans élections, et le système judiciaire reposerait uniquement sur la chariat, interprétée par les oulémas. On voit donc à quel point le système est différent de l'Iran par exemple, où il existe une représentation parlementaire où les oulémas ne sont pas dominants et une palette de mouvements politiques. Les oulémas à la tête des Taléban visent directement l'instauration d'un pouvoir théocratique. Pour prendre une comparaison historique, en 1929, lors de la révolte contre le programme de modernisation initié par le roi Amanoullah, les oulémas n'avaient pas essayé de prendre le pouvoir, même s'ils avaient été un élément clé de l'installation d'une nouvelle dynastie. Le retour du roi Zaher, qui semble refusé par la plupart des Taléban (et comment articulé les pouvoir de l'amir et du roi ?), montre l'évolution sociale de ces dernières décennies.

Les deux réponses au désordre : oulémas contre éduqués.

14 La prise d'Hérat, puis de Kaboul, s'est faite militairement, sans soutien notable de la population ${ }^{44}$. Après une percée initiale rapide, les Taléban se sont engagés dans des opérations où les facteurs de succès tiennent d'abord à l'efficacité de l'appareil militaire et aux dissensions de l'ennemi. En effet, l'avancée des Taléban s'est rapidement heurtée à des systèmes politiques régionaux constitués qui étaient sortis de la fragmentation initiale. Dans les régions qui connaissaient une situation de paix, 
l'accueil fait aux Taléban a été assez hostile : la population d'une ville comme Hérat a globalement perdu en sécurité et en bonne administration avec l'arrivée des Taléban. Ceux-ci ne sont donc pas une réponse universelle à une situation générale de désordre. La situation n'est pas la même à Qandahar que dans les zones relativement bien administrées d'Hérat ou de Mazar. Les deux évolutions politiques divergentes par rapport à la fragmentation initiale ont eu pour résultat une territorialisation politique qui accentue les différences régionales et conduit à une dichotomie nord-sud, qui est initialement sociale avant d'être régionale-communautaire. Les groupes sociaux se distinguent ici en quelques oppositions; citadins (et particulièrement kaboulis) contre ruraux, oulémas contre laïques, éduqués contre non-éduqués et tribalisés contre nontribalisés, qui permettent de comprendre les réactions différentes par rapport aux Taléban. L'appartenance à ces groupes sociaux est plus prédictive des préférences politiques que l'appartenance linguistique ou ethnique, même si l'interprétation communautaire du conflit est utilisé par certains acteurs pour décrire le conflit. La pratique des Taléban, au-delà des discours universalistes, est marquée par leur recrutement majoritairement pashtoune. Les Taléban ont également des réflexes communautaires marques. Ainsi, après la prise de Kaboul, ont-ils pris en otage plusieurs centaines de panjshiris de la capitale pour les échanger contre leurs soldats faits prisonniers par Massoud lors de leur opération ratée dans le Panjshir. Au moment où Ismael Khan menaçait sérieusement Qandahar, les Taléban ont eux-mêmes joué sur un réflexe communautaire en accusant Ismael Khan d'être un nationaliste tadjik, qui se livrerait aux pires horreurs s'il prenait la ville. Le mouvement est perçu ailleurs comme ethnique (reflétant la culture pashtoune, la domination historique de cette ethnie sur le système politique, d'où le ralliement de certains royalistes aux Taléban). La prise d'otages panjshiri à Kaboul pour être échangé contre les Taléban capturés par Massoud montre bien l'interprétation communautaire qui est la leur. Enfin, la dynamique actuelle de polarisation exclut tout compromis. Les perspectives de conciliation sont d'autant plus faibles que le maintien des hostilités est nécessaire à la cohésion des deux camps. Au nord, l'alliance entre éduqués (islamistes et ex-communistes) et oulémas chi'ites est idéologiquement incohérente et une diminution de la pression militaire des Taleban la ferait probablement éclater. La coalition du nord ne repose que sur l'urgence de la survie, sans projet politique commun. Le Jamiat, réduit à une dimension régionale après la perte d'Hérat et de Kaboul, ne peut plus jouer le rôle de pivot d'une coalition, alors que le Djoumbesh, force militaire dominante, suscite des réactions de rejet en fonction de son origine. Au sud, l'administration des Taleban crée une l'insatisfaction chez les éduques et les citadins en général, mais rien ne laisse présager un éclatement, même si le mouvement peut avoir du mal à gérer les appartenances tribales et locales. Pour leur part, les Taléban ont besoin de la dynamique de guerre pour canaliser les tensions entre les différents réseaux internes au mouvement. Leur légitimité est également liée à la rupture avec les partis et les commandants et donc le refus de passer des accords qui, de façon très probable, ne seraient que provisoires. L'avenir immédiat de l'Afghanistan est donc entre l'ordre puritain des oulémas et le projet de modernisation avorté des éduqués.

Résumé de l'article. 


\section{NOTES}

1. Notamment Olivier Roy, Afghanistan, Islam et modernité politique, Seuil-Esprit, 1985.

2. Ce qu'ont sait aujourd'hui du processus de décision ayant conduit à l'invasion soviétique met surtout en évidence l'épuisement de Brejnev, alcoolique et dépressif, et le poids du dogme marxiste sur l'irréversibilité du socialisme, plus qu'un projet d'accès aux mers chaudes.

3. La thématique du Grand Jeu, l'affrontement entre l'Empire russe et britannique au siècle dernier, est particulièrement fréquent, voir Mike Barry, Le royaume de l'insolence, ;

4. Cet échec s'explique par l'importance du soutien soviétique àprès 1989 et la stratégie inadaptée des moudjahiddin sous influence américano-pakistanaise.

5. Voir l'article d'Asian Survey.An hobesian state of nature et renvoi à l'article de La Lettre d'Asie Centrale.

6. Hérodote, printemps 1997.

7. Voir Current History.

8. Voir Lettre d'Asie centrale.

9. Plusieurs milliards de dollars essentiellement donnés par les Etats-Unis et les pays arabes du Golfe.

10. Des systèmes intermédiaires sont envisageables comme la rotation des frères.

11. Parmi les grands commandants assassinés par des groupes concurrents, mollah Nassim, Zabihoullah, Saddeqi de Nili, Safihoullah Afzali, Ismael Tariq...

12. Centlivres, Seurat et Carré.

13. Olivier Roy.

14. Numéro spécial sur la vendetta voir dossier Turquie.

15. Référence sur l'incident et réaction des FM.

16. Le passage qui suit reprend en partie un article paru dans Afghanistan Info, $n^{\circ} 31$, mars 1992.

17. En effet, le Harakat était organisé autour du commandant mohallem Razorq, un instituteur réputé pour son sens de l'organisation. Il s'est heurté violemment au commandant Abdul Haï, également à la tête d'un groupe important du Harakat, en 1984 les combats firent une quarantaine de morts.

18. Le commandant Yâcin, un Laghmani persanophone d'une quarantaine d'années rassemble des petits boutiquiers du bazar, avec l'appui des oulémas traditionnels.

19. Pour une description de cette situation voir mon article dans Afghainfo.

20. Voir Les Nouvelles d'Afghanistan.

21. Source, C'est ce qui explique en partie le passage de nombreux Hezb-é islami aux Taleban.

22. Référence Shahrani.

23. Voir Charles Lindholm, Frontier Perspectives, Essays in comparative Anthropology, Oxford, Oxford University Press, 1996.

24. Voir ce qu'en dit Olivier Roy au début des années quatre-vingt.

25. Voir And your name shall rise.

26. Voir notre article dans Cultures et Conflits. 
27. On peut définir les éduqués comme le groupe passé par le lycée ou l'Université, et dans lequel se recrute la majorité des militants politiques d'avant 1978.

28. Dès le début de la guerre, l'affiliation des très nombreux groupes de combattants se fait largement au hasard (beaucoup de moudjahiddin ne savent pas à quel parti ils appartiennent en théorie). Jusqu'en 1989, les conflits sont limités par le poids des oulémas qui imposent des arbitrages et par la présence soviétique qui joue le rôle d'unificateur.

29. Interview avec le pir automne 1992.

30. L'intégration des jeunes des milieux populaires urbains dans les futtuwa est une création du calife Nasr, les paylouch en sont peut-être une survivance Ashtor E., A social and Economic History of the Near Middle East in the Middles Ages, Londres, 1976, page 234 .

31. Il y a un rapport plus que géographique entre ces trois activités : la visite du ziarat, fumer du haschisch et les combats de chiens. On peut dire, en forçant un peu le trait, que le style de la ville de Qandahar s'exprime parfaitement ici, à ceci près qu'il faudrait ajouter la musique, omniprésente dans la ville (jusqu'à l'arrivée des Taleban).

32. Cette affirmation est vraie pour les ruraux, dans le cas des urbains, des nuances sont à faire, en dehors même des militants communistes, en nombre restreint, il y avait aussi des groupes gauchistes (maoïstes notamment), qui ont pu trouver quelques appuis dans des zones rurales.

33. Le jihad est un acte volontaire, alors que la prière, par exemple, est obligatoire, et il suffit qu'un nombre suffisant de fidèles participent à la défense de la communauté.

34. Sur la notion de shahid voir Charnay, Sociologie religieuse de l'islam, Hachette, 1994, Paris, p 252 et suivantes.

35. Dans les régions pashtounes tribalisées, le modèle du guerrier recoupe celui du moudjahidine. Cependant, la notion de moudjahidine est plus légitimante que celle du guerrier, qui obéit au code tribal pashtoune.

36. Pierre Centlivres, op cit page 58 .

37. Du nom de l'émir mis sur le trône par les Britanniques en 1839, et devenu symbole de la collaboration avec l'impérialisme étranger.

38. Le rôle des services secrets afghans, Khâd, est ici central. Leur budget aurait été à peu près l'équivalent de celui de l'Etat.

39. Voir le numéro spécial de La Lettre d'Asie centrale consacrée au wahabbisme.

40. Il n'y a pas eu d'enquête systématique sur ce thème, cette impression est tirée de nombreuses conversations depuis quelques années.

41. Le transport notamment a généralement été assuré par des privés contre paiement mais, dans les premières années, la nourriture, les services d'un guide... étaient le plus souvent fournis gratuitement par les paysans, au nom du jihad.

42. Sur la problématique de l'intervention pakistanaise et du soutien américain.

43. Importance du concept, Opposition de principe à a pluralité.

44. Dans le cas de Djalalabad, où il n'y avait précisemment pas de stabilité politique, des témoignages concordant montrent le fort soutien populaire aus Taleban. 
INDEX

Index géographique : Afghanistan

Mots-clés : crises politiques, guerre, sciences politiques

\section{AUTEUR}

\section{GILLES DORRONSORO}

Cette étude est basée sur un travail de terrain effectué en décembre-janvier 1997. Je remercie l'EHESS, qui a pris en charge une partie des frais, et les associations humanitaires qui m'ont hébergé pendant mon séjour, notamment, AMI (Aide médicale internationale), HI (Handicap International) et MSF (Médecins sans frontière). 